\title{
Preparation and characterization of carbamoylethyl hemicelluloses
}

\author{
Jun-Li Ren, ${ }^{1,3}$ Feng Peng, ${ }^{1}$ Run-Cang Sun, ${ }^{1,2^{*}}$ Xun-Li Zhang ${ }^{4}$ \\ ${ }^{1 *}$ State Key Laboratory of Pulp and Paper Engineering, South China University of \\ Technology, Guangzhou, 510640, China; tel: +86-20-87111861; e-mail: \\ ynsun@scut.edu.cn \\ ${ }^{2}$ College of Material Science and Engineering, Beijing Forestry University, Beijing, \\ 100083, China; tel: +86-10-62336972; e-mail: rcsun3@bjfu.edu.cn \\ ${ }^{3}$ Key Laboratory of Cellulose and Lignocellulosics Chemistry, Guangzhou Institute of \\ Chemistry, 510650, China; tel: +86-20-85231445; e-mail: junliren2008@hotmail.com \\ ${ }^{4}$ School of Engineering Sciences, University of Southampton, Southampton SO17 \\ 1BJ, UK; tel: +44-23-80598748; fax: +44-23-8059 3016; e-mail: XL.Zhang@ \\ soton.ac.uk
}

(Received: 16 June, 2008; published: 31 January, 2009)

\begin{abstract}
The reaction of hemicelluloses, isolated from wheat straw, with acrylamide in alkaline aqueous solution was investigated. The degree of substitution (DS, up to 0.65 ) of the products could be obtained by adjusting the molar ratios of reagent to xylose unit in hemicelluloses (1.0-10.0) and the molar ratios of catalyst to xylose unit in hemicelluloses (0.5-1.6). Changes in the structure of hemicelluloses were verified by FT-IR and ${ }^{1} \mathrm{H}$ and ${ }^{13} \mathrm{C}$ NMR spectroscopies. The results showed that carbamoylethyl groups surely were grafted onto the main chain of hemicelluloses. Moreover, it was also found that the saponification of carbamoylethyl groups to carboxyl groups occurred in alkaline medium. The behavior of modified hemicelluloses was monitored by means of thermo gravimetry (TG) and differential thermal analysis (TGA). It was found that the products had a lower thermal stability than the native hemicelluloses.
\end{abstract}

\section{Introduction}

Sustainable sources of materials to supply the needs of society in the coming decades are much needed at present as the world becomes increasingly aware of the limited nature of fossil fuels. In response to this situation, lignocellulosic biomass from trees, grasses, cereals, and other plants, has become the main focus of the developing biorefining industry [1]. Hemicelluloses, the second most abundant class of polysaccharides found in nature after cellulose, recently gain increasing importance as basis for new biopolymeric materials and functional polymers accessible by chemical modification reactions [2]. From the chemist's point of view a brood variety of chemical modification reaction both at the $\mathrm{OH}$ groups and the $\mathrm{C}$ atoms are possible [3]. Thus, the reactive hydroxyl groups are accessible to typical conversions of the $\mathrm{OH}$ moieties like esterificaion and etherification, and nucleophilic displacement reactions. At present, modification of hemicelluloses with unconventional functional groups is of increasing interest. Thus, various novel hemicellulosic derivatives with different degree of substitution (DS) were synthesized and characterized, e.g., crown ether containing hemicelluloses ethers $[4,5,6]$ or crown ester containing hemicelluloses ester [7-10]. 
Wheat straw contains $14-15 \%$ lignin, $35-40 \%$ cellulose, and $30-35 \%$ hemicelluloses [11]. The hemicelluloses are made up of a $(1 \rightarrow 4)$ linked $\beta$-D-xylan main chain with Larabinofuranosyl and D-xylopyranosyl side chains attached at position O-C (3), and D-glucopyranosyluronic acid (or 4-O-methyl-D-glucopyranosyluronic acid) groups attached at position O-C (2) [12]. The backbone contains $\beta$-D-xylopyranose units, each of which has two hydroxyl groups available for modification. The hydroxyl groups allow the potential for esterification, etherification, oxidation and other reactions such as hydrolysis and reduction. Thus, the polymer contains hydroxyl groups and offers the possibility to obtain amphoteric products by reacting with acrylamide based on the Michael addition. Up to now no papers about this reaction of hemicelluloses was published. The aim of this research is a preliminary study of the influence of the conditions under which the reaction takes place (molar ratio of catalyst to xylose unit in hemicelluloses and molar ratio of reactants to xylose unit in hemicelluloses) on degree of etherification of the products obtained. The products were characterized regarding structure and thermal properties.

\section{Results and discussion}

\section{Analysis of the isolated native hemicelluloses}

On the basis of the dry weight of wheat straw, treatment of the delignified holocellulose with $10 \% \mathrm{KOH}$ at $23{ }^{\circ} \mathrm{C}$ for $10 \mathrm{~h}$ yielded $34.3 \%$ alkali-soluble hemicelluloses. The sugar analysis showed the sugar composition (relative $\%$ ): $82.3 \%$ xylose, $9.6 \%$ arabinose, $4.0 \%$ glucose, $2.4 \%$ galactose, $0.7 \%$ rhamnose, $0.8 \%$

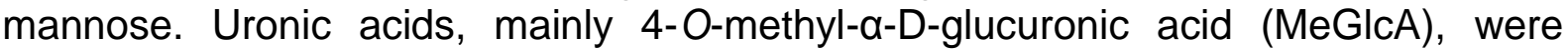
present in a noticeable amount (3.9\%).

\section{Synthesis of carbamoylethyl hemicelluloses in aqueous alkaline solution}

Usually, vinyl monomers can be grafted onto cellulose or starch by graft copolymerization through various methods. However, acrylonitrile and acrylamide can also be served as etherification reagents and reacted with cellulose and starch in an alkaline medium $[13,14,15]$. Xylose, which accounted for $82.3 \%$ in sugar composition, is the main component in wheat straw hemicelluloses, and xylose unit in hemicelluloses have two active hydroxyl groups, which allow the potential for etherification. In a Michael addition, acrylamide can be added to hemicelluloses with formation of the carbamoylethyl ether of hemicelluloses.

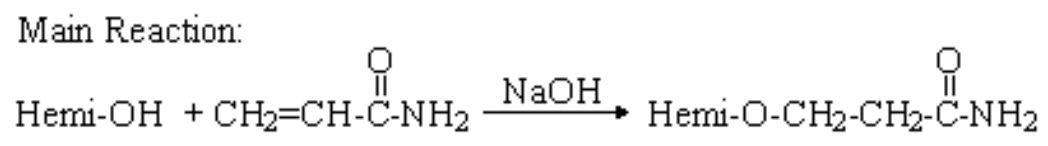

Side Reaction:

Hemi-O- $\mathrm{CH}_{2}-\mathrm{CH}_{2}-\mathrm{C}-\mathrm{NH}_{2} \stackrel{\mathrm{NaOH}}{\longrightarrow}$ Hemi-O-CH $2-\mathrm{CH}_{2}-\mathrm{C}-\mathrm{ONa}+\mathrm{NH}_{3}$<smiles>C=CC(N)=O</smiles>

Scheme 1. The etherificaition of hemicelluloses with acrylamide in alkaline aqueous solution. 
Moreover, the acylamino groups can be easily saponified to a carboxyl group to give carboxyethyl polymer as the stable end-product in a higher alkaline aqueous medium at high temperature [16]. Scheme 1 illustrates the etherification of hemicelluloses in alkaline aqueous solution, and the reaction conditions are listed in Tab.1. To keep the side reaction from happening, the reaction performed at $25^{\circ} \mathrm{C}$ for $12 \mathrm{~h}$. The optimization of the process of etherification was performed by varying the process of parameters such as the molar ratio of sodium hydroxide to xylose unit in hemicelluloses and the molar ratio of acrylamide to xylose unit in hemicelluloses. DS was calculated based on the assumption that hemicelluloses were all xylose, so the maximum DS is 2.The DS of the products could be determined from the peak area ratio of hydrogen in C-7 to the hydrogen in xylose unit. Tab. 1 shows the effect of molar ratio of sodium hydroxide to xylose unit in hemicelluloses from 0.5 to 1.6 on DS. It was observed that DS of products increased from 0.29 to 0.50 as the molar ratio of $\mathrm{NaOH}$ to xylose unit in hemicelluloses increased from 0.5 to 1.6 , indicating that the catalyst of the $\mathrm{NaOH}$ could increase the reaction rate. At high alkaline concentration saponification are predominant in this reaction, and more carboxyl groups were formed, which resulted in increase of DS of carboxyethyl groups. This phenomenon should be avoided. Furthermore, the effect of the molar ratio of acrylamide to xylose unit in hemicelluloses on DS is shown in Tab. 1 from samples 6 to 10 and 3 . As can been seen, a maximum DS of 0.65 was obtained with the molar ratio of acrylamide to xylose unit in hemicelluloses of 8.0. There was an increase in the DS with the molar ratio from 1.0 to 8.0 and thereafter it decreased with the molar ratio from 8.0 to 10.0 . The increase in the DS of resulted products was probably due to the greater availability of acrylamide at higher concentration in the proximity of hemicelluloses molecules. The decrease in the DS may be explained by that a sufficient time may not have been given for large extend of etherification at high AM concentrations. In addition, except for samples 6 and 7, other samples showed good solubility in water. Because carbamoylethyl groups of the substituent were partially saponified to carboxyethyl groups, the derivatives displayed the typical solution behavior of anionic polyelectrolytes.

Tab. 1. Degree of substitution (DS) of carbamoylethyl hemicelluloses obtained by the etherification of hemicelluloses with acrylamide in alkaline aqueous solution.

\begin{tabular}{ccccc}
\hline $\begin{array}{c}\text { Sample } \\
\text { No }\end{array}$ & Molar ratio $^{\mathrm{a}}$ & Molar ratio $^{\mathrm{b}}$ & $\begin{array}{c}\text { Solubility in } \\
\text { water }^{\mathrm{c}}\end{array}$ & $\begin{array}{c}\text { Degree of } \\
\text { substituent }^{\mathrm{D}}\end{array}$ \\
\hline 1 & 0.5 & 4.0 & + & 0.29 \\
2 & 0.8 & 4.0 & + & 0.34 \\
3 & 1.0 & 4.0 & + & 0.44 \\
4 & 1.2 & 4.0 & + & 0.45 \\
5 & 1.6 & 4.0 & + & 0.50 \\
6 & 1.0 & 1.0 & - & 0.15 \\
7 & 1.0 & 2.0 & - & 0.25 \\
8 & 1.0 & 6.0 & + & 0.51 \\
9 & 1.0 & 8.0 & + & 0.65 \\
10 & 1.0 & 10.0 & + & 0.58 \\
\hline
\end{tabular}

\footnotetext{
${ }^{\text {a }}$ Represent the molar ratio of sodium hydroxide to xylose unit in hemicelluloses, xylose unit $M_{w}=132$.

${ }^{\mathrm{b}}$ Represent the molar ratio of acrylamide to xylose unit in hemicelluloses, xylose unit $M_{w}=132$.

${ }^{c}$ + soluble, - not completely soluble.

${ }^{\mathrm{d}}$ Determined by ${ }^{1} \mathrm{H}$ NMR.
} 


\section{FT-IR spectra}

FT-IR spectra of native hemicelluloses (spectrum a) and modified hemicelluloses (sample 3, spectrum b) are shown in Fig. 1. The spectra had a specific band maximum in the $1200-1000 \mathrm{~cm}^{-1}$, which are typical of xylans. This region is dominated by ring vibrations overlapped with stretching vibration of $(\mathrm{C}-\mathrm{OH})$ side groups and the (C-O-C) glycosidic bond vibration. In particular, the band between 1108 and $1030 \mathrm{~cm}^{-1}$ is dominated by glycosidic linkages (C-O-C) contribution [17]. In the carbonyl stretching region, a strong band at $1607 \mathrm{~cm}^{-1}$ is assigned to the absorbed water. The band at $891 \mathrm{~cm}^{-1}$, which represents the $\mathrm{C}-1$ groups frequency or ring frequency, is characteristic of $\beta$-glycosidic linkages between the sugar units [18]. The bands between 1466 and $1246 \mathrm{~cm}^{-1}$ arise from $\mathrm{C}-\mathrm{H}, \mathrm{OH}$ or $\mathrm{CH}_{2}$ bendings. A strong broad band due to hydrogen-bonded hydroxyl appears at $3410 \mathrm{~cm}^{-1}$ and the symmetric $\mathrm{C}-\mathrm{H}$ vibration band at $2923 \mathrm{~cm}^{-1}$. Compared with the spectrum a, the spectrum $b$ of modified hemicelluloses sample 3 provides evidence of etherification by an increment in the intensity of the major ether bands at $1042 \mathrm{~cm}^{-1}$. Furthermore, the new band at $1666 \mathrm{~cm}^{-1}$ for the derivatives represent amide I $(C=O)$ bands. Another two new peaks at 1610 and $1403 \mathrm{~cm}^{-1}$ are observed due to the asymmetrical and symmetrical stretching of $-\mathrm{COO}^{-}$groups, respectively. Those new bands confirmed that carbamoylethyl groups undoubtedly were be grafted onto the backbone of hemicelluloses, and additionally carboxyl groups shown in spectrum b above indicated that saponification happened in alkaline condition.

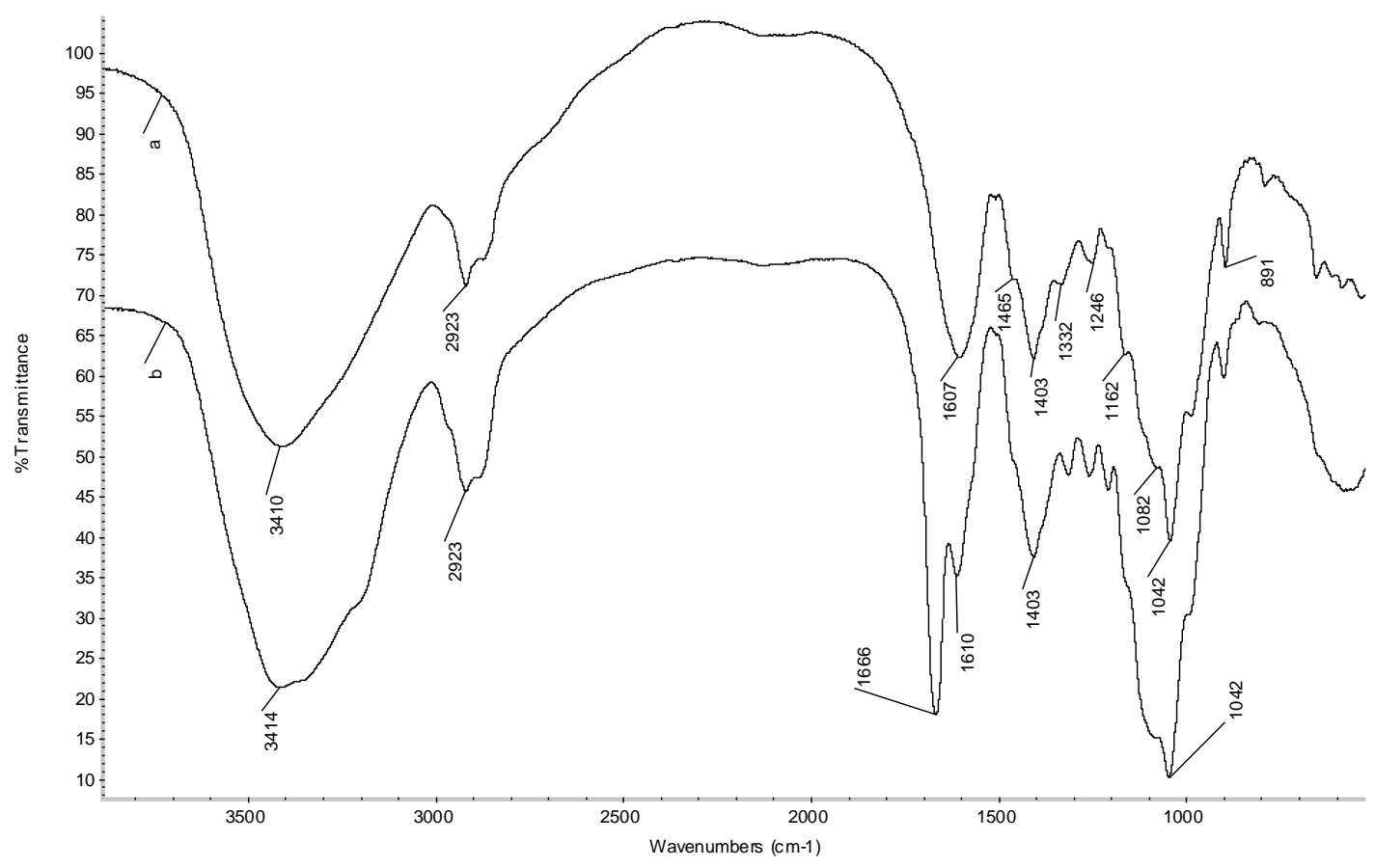

Fig. 1. FT-IR spectra of native hemicelluloses and modified hemicelluloses sample 3.

Fig. 2 illustrates FT-IR spectra of modified hemicelluloses prepared under the molar ratio of $\mathrm{NaOH}$ to xylose unit in hemicelluloses of 0.5 (spectrum a, sample 1), 1.0 (spectrum b, sample 3), and 1.2 (spectrum c, sample 4). Comparing the relative intensity at 1666 and $1605 \mathrm{~cm}^{-1}$, the spectra showed an increase in the intensity with an increment of the molar ratio of sodium hydroxide to xylose unit in hemicelluloses from 0.5 to 1.0 , and to 1.2 , corresponding to the increase of DS values from 0.29 
(spectrum a) to 0.44 (spectrum b), and to 0.45 (spectrum c). While an increment in the intensity of these peaks at 1605 and $1405 \mathrm{~cm}^{-1}$ (the asymmetrical and symmetrical stretching of $-\mathrm{COO}^{-}$groups) with an increment of the molar ratio of $\mathrm{NaOH}$ to xylose unit in hemicelluloses, indicated that more carbamoylethyl groups were saponified to carboxyethyl groups at higher alkaline aqueous medium, which was not expected.

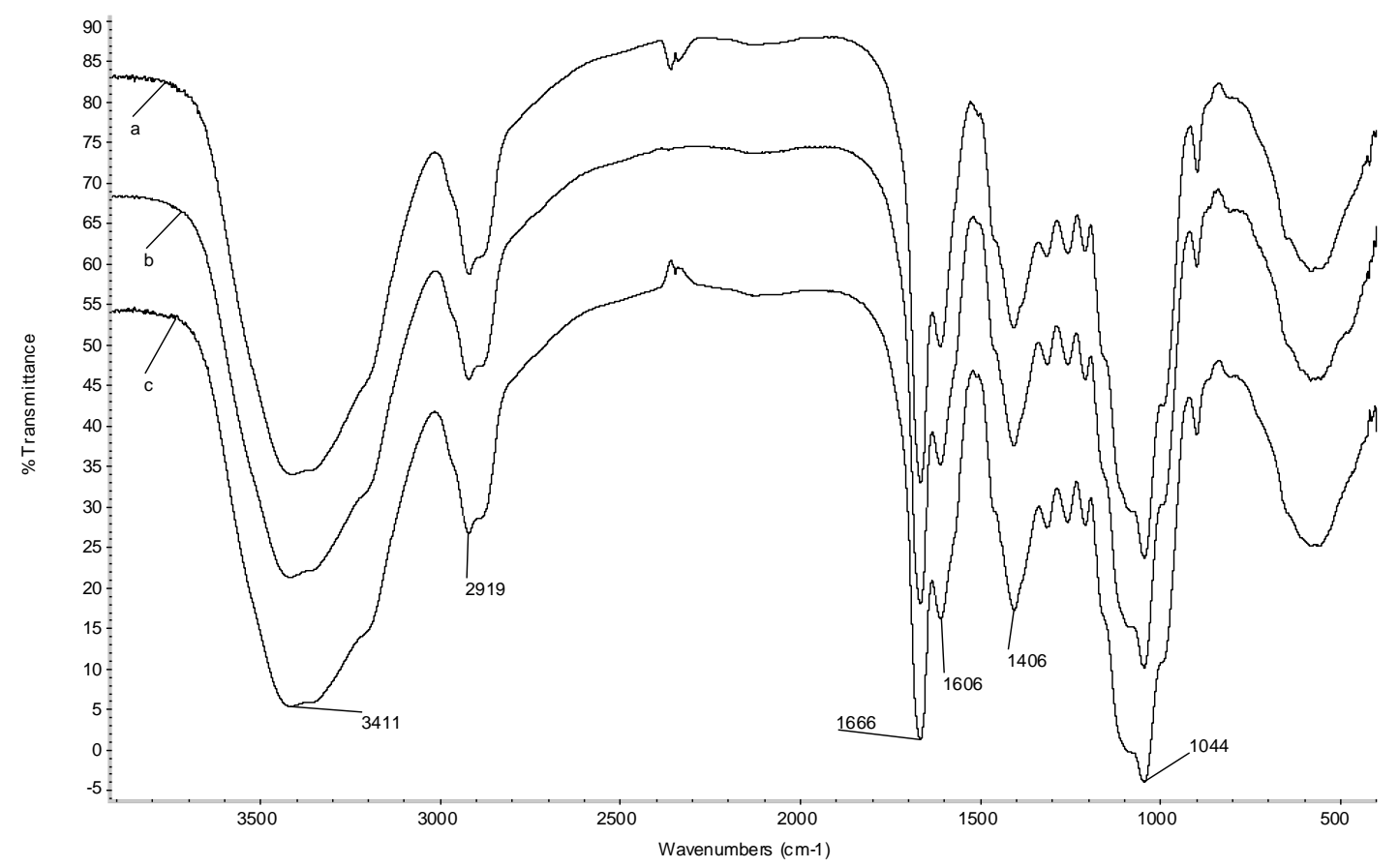

Fig. 2. FT-IR spectra of modified hemicelluloses samples 1 (spectrum a), 3 (spectrum b), and 4 (spectrum c).

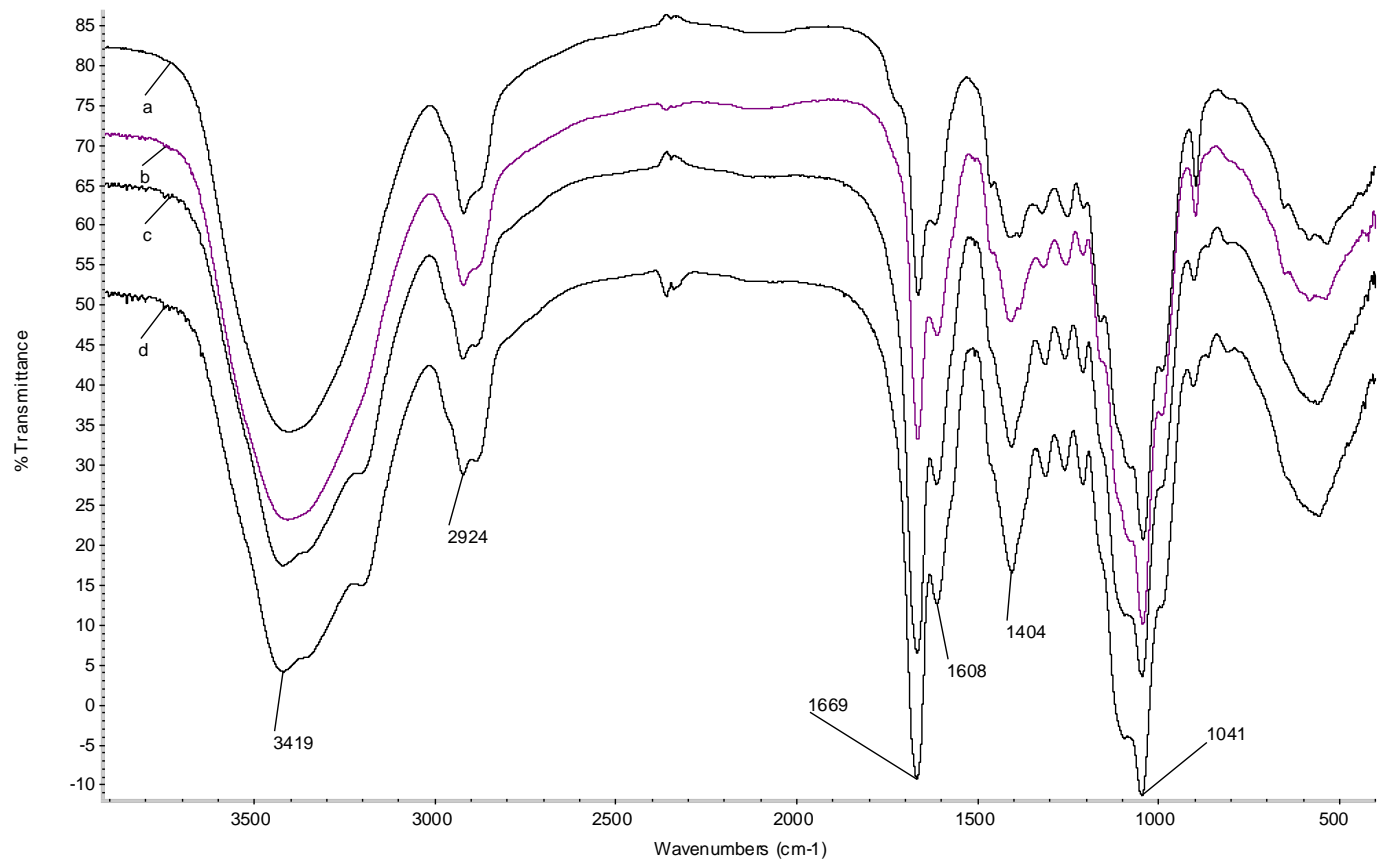

Fig. 3. FT-IR spectra of modified hemicelluloses sample 6 (spectrum a), sample 7 (spectrum b), sample 10 (spectrum c), and sample 9 (spectrum d). 
Fig. 3 shows FT-IR spectra of modified hemicelluloses synthesized at the molar ratio of acrylamide to xylose unit in hemicelluloses of 1.0 (spectrum a, sample 6), 2.0 (spectrum b, sample 7), 8.0 (spectrum d, sample 9), and 10.0 (spectrum c, sample 10). Obviously, an increase in the molar ratio of acrylamide to xylose unit in hemicelluloses from 1.0 (sample 6), to 2.0 (sample 7), to 8.0 (sample 9), and to 10.0 (sample 10) resulted in an increase in the intensity of the peaks at 1669 and 1608 $\mathrm{cm}^{-1}$ from spectrum a (sample 6), to spectrum b (sample 7), and to spectrum d (sample 9), and then a decrease in the intensity from spectrum d (sample 9) to spectrum c (sample 10), corresponding to an increase in DS from 0.15 (sample 6) to 0.25 (sample 7), and to 0.65 (sample 9), and then a decrease in DS from 0.65 (sample 9) to 0.58 (sample 10). This is in agreement with the description of the influence of the molar ratio of acrylamide to xylose unit in hemicelluloses on DS of products in Tab. 1.

\section{${ }^{1} \mathrm{H} N M R$ and ${ }^{13} \mathrm{C} N \mathrm{MR}$ spectra}

Fig. 4 shows ${ }^{1} \mathrm{H}$ NMR spectra of native hemicelluloses (spectrum a) and modified hemicelluloses sample 2 (spectrum b). A strong signal at $4.7 \mathrm{ppm}$ is originated from the residual solvent (HDO). The chemical shifts of 3.2-4.4 ppm arise from the equatorial proton and other protons of anhydroxylose units of hemicelluloses. The methyl protons of few amounts of acetyl group exhibit peaks at $1.8 \mathrm{ppm}$. Anomeric protons of terminal $\alpha-D$-arabinofuranosyl residues give a shoulder at $5.2 \mathrm{ppm}$ [19] in spectrum a.

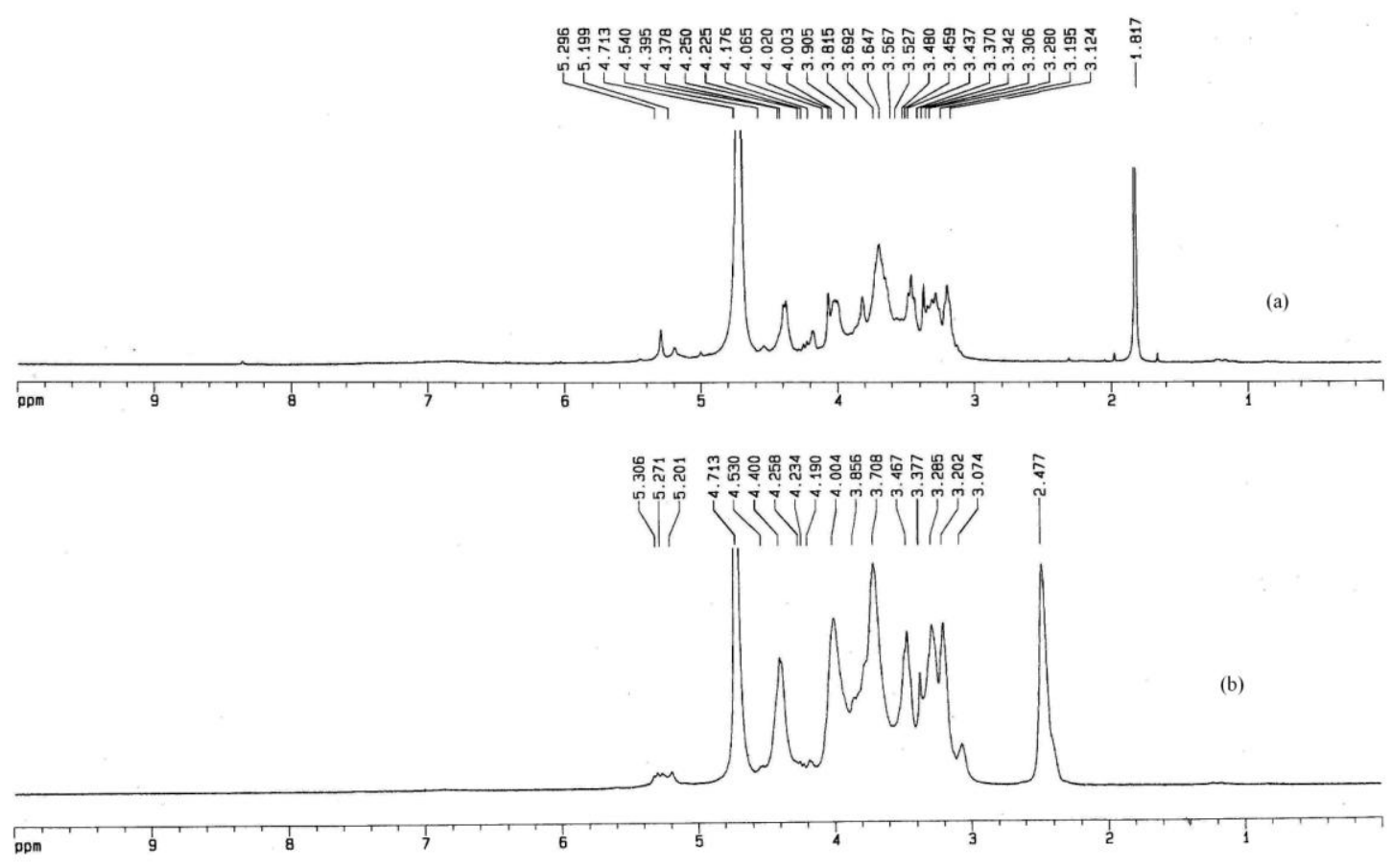

Fig. 4. ${ }^{1} \mathrm{H}$ NMR spectrum of native hemicelluloses (spectrum a) and modified hemicelluloses sample 2 (spectrum b). 
Furthermore, ${ }^{1} \mathrm{H}$ NMR spectra of modified hemicelluloses sample 2 is shown in spectrum b of Fig. 4. Compared with native hemicelluloses, there exists a new broad peak at $2.4 \mathrm{ppm}$ in spectrum $\mathrm{b}$, which is assigned to protons of methylene connected to carbamoylethyl groups and carboxyethyl groups. This new peak confirmed that this etherification reaction occurred on hemicelluloses macromolecule.

The structure of the hemicelluloses and modified hemicelluloses sample 2 were revealed by ${ }^{13} \mathrm{C}$ NMR spectroscopy in Figs. 5 and 6 , respectively. Most of the major resonances were assigned by references to data in literature $[20,21]$ The main 1,4linked $\beta$-D-Xylp units are obviously characterized by five strong signals at 101.6, 76.2, 73.6, 72.6, $62.9 \mathrm{ppm}$, which are assigned respectively to C-1, C-4, C-3, C-2, and C-5 position of the $\beta$-D-Xyl units. The signals at 108.4, 84.5, 80.7, 77.2, and 61.2 ppm correspond to C-1, C-4, C-2, C-3, and C-5 of $\alpha$-L-arabinofuranosyl residues to $\beta$ D-xylans. Two signals at 71.1 and $68.4 \mathrm{ppm}$ represent C-4 and C-2 of galactose residue in the xylan. The peaks at $82.4 \mathrm{ppm}$ and $57.4 \mathrm{ppm}$ are originated from C-4 and methoxyl group of a 4-O-methy-D-glucuronic acid residue in the xylan. Acetyl $\mathrm{CH}_{3}$ in xylan gives a signal at $23.2 \mathrm{ppm}$. The presence of quantities of associated lignin was identified by one weak signal at $181.5 \mathrm{ppm}$, which originates from the carbonyl group in associated lignin.

Fig. 6 shows the ${ }^{13} \mathrm{C}$ NMR spectra of modified hemicelluloses sample 2 in $\mathrm{D}_{2} \mathrm{O}$ at 25 ${ }^{\circ} \mathrm{C}$. The chemical shift at 177.0 and $180.1 \mathrm{ppm}$ are assigned to the carbon signal of carbonyl groups in carbamoylethyl (C-8) and carboxyethyl (C-8') groups [22,23], respectively. Doublet at 35.7 and $38.2 \mathrm{ppm}$ are attributed to methylene that connect to carbamoylethyl (C-7) and carboxyethyl (C-7') groups, respectively. The peaks for hemicelluloses backbone and another methylene (C-6, C- $\left.6^{\prime}\right)$ of the substituents are between 60 and $105 \mathrm{ppm}$. Moreover, The DS of carbamoylethyl and carboxyethyl groups could be obtained according to the peak intensity of the two carbonyl peaks or the methylene peaks that connect to carbamoylethyl and carboxyethyl groups, and the results illustrated that DS carbamoylethyl groups was 0.51 and DS carboxyethyl groups was 0.14 . So these new signals verified that hemicelluloses were etherified to carbamoylethyl hemicelluloses.

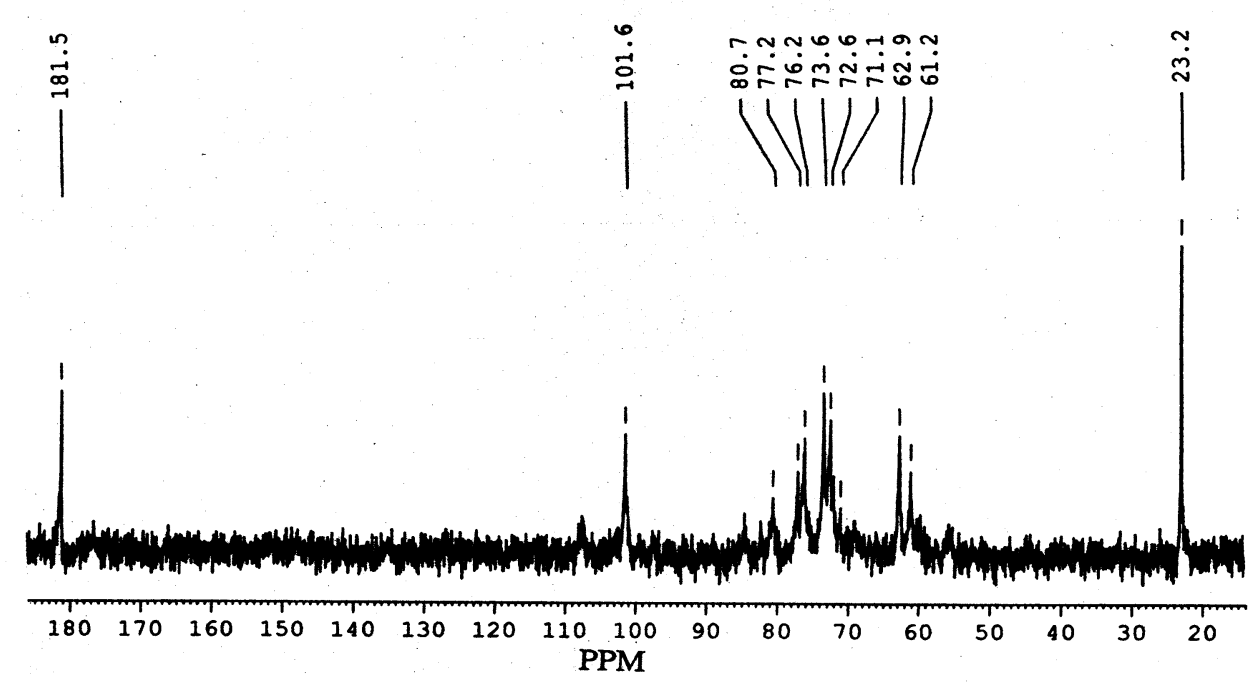

Fig. $5 .{ }^{13} \mathrm{C}$ NMR spectrum of native hemicelluloses in $\mathrm{D}_{2} \mathrm{O}$ at $25^{\circ} \mathrm{C}$. 


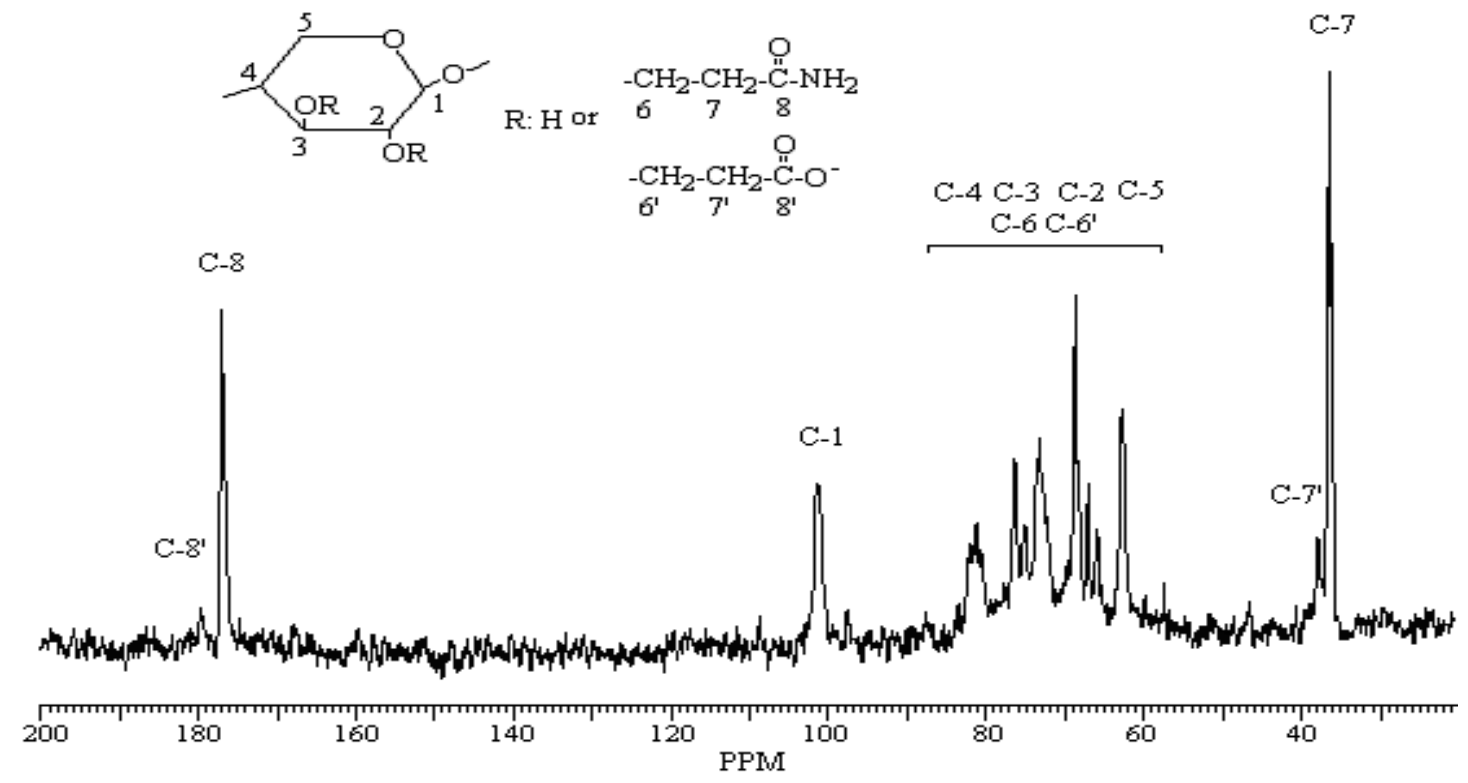

Fig. 6. ${ }^{13} \mathrm{C}$ NMR spectrum of modified hemicelluloses sample 2 in $\mathrm{D}_{2} \mathrm{O}$ at $25{ }^{\circ} \mathrm{C}$.

\section{Thermal analysis}

The results obtained from the typical TG/DTA curves of modified hemicelluloses sample 4 and native hemicelluloses are given in Fig. 7. Clearly the weight loss between 40 and $150{ }^{\circ} \mathrm{C}$ was due to the loss of water naturally trapped into the polymer.

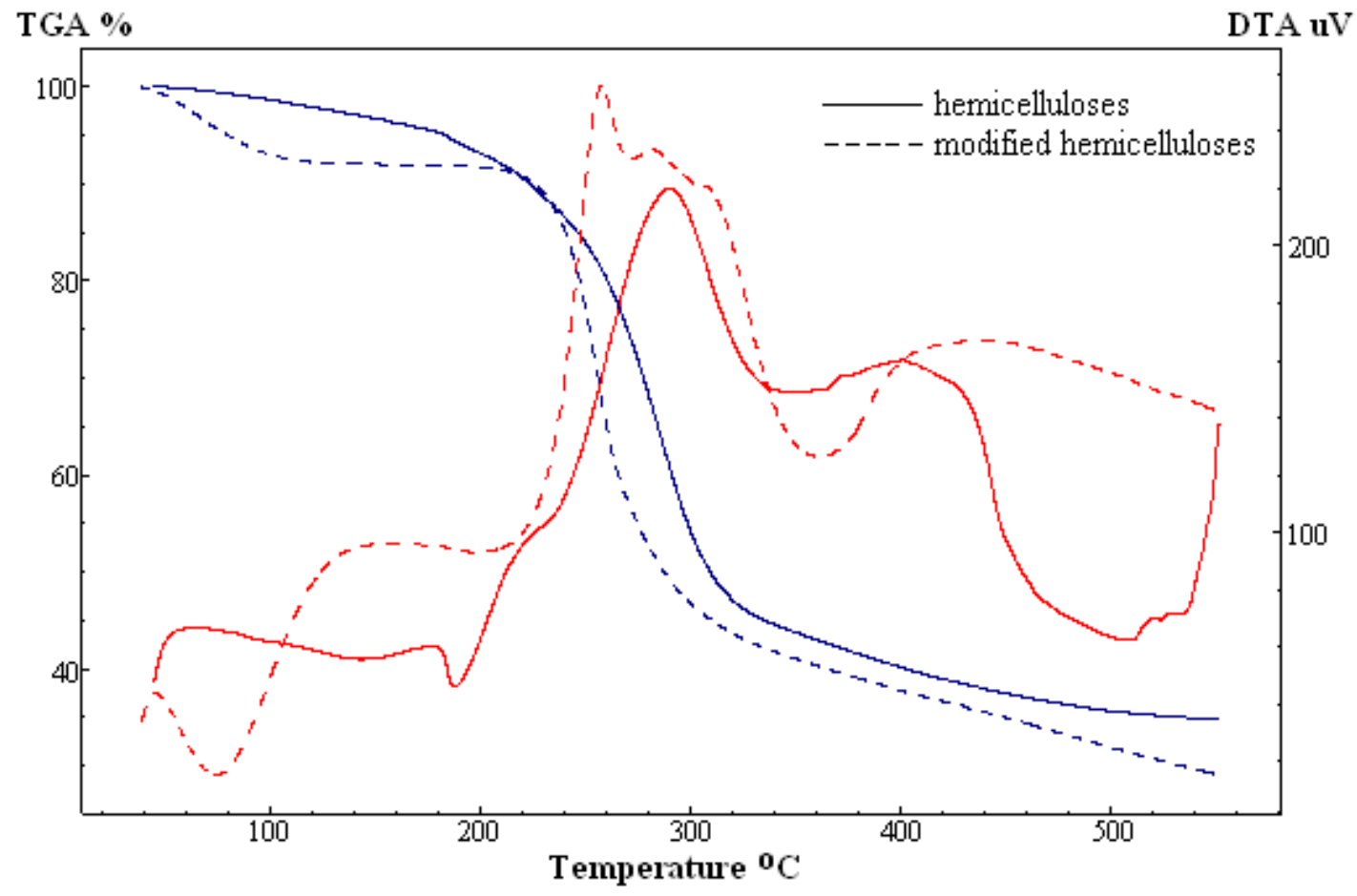

Fig. 7. Thermograms of modified hemicelluloses sample 4 and native hemicelluloses. 
The native hemicelluloses and modified hemicelluloses sample 4 started to decompose at 223 and $214{ }^{\circ} \mathrm{C}$, and the weight loss of $50 \%$ occurred at 312 and 286 ${ }^{\circ} \mathrm{C}$, respectively. This indicated that the native hemicelluloses were more stable than the modified hemicelluloses. In other words, the thermal stability of the unmodified hemicelluloses was higher than that of modified hemicelluloses, which was probably due to the disintegration of the intramolecular interactions such as hydrogen bonds between the polymer chains under chemical modification and the form of the products with unstable carbamoylethyl groups. In DTA curves, the exothermic peaks occurred at 289 and $404{ }^{\circ} \mathrm{C}$ for the native hemicelluloses and at 214 and $420{ }^{\circ} \mathrm{C}$ for the modified hemicelluloses. That indicated again that the thermal stability of modified hemicelluloses decreased because of the increment of molar mass of polymer ethers and carbamoylethyl groups.

\section{Conclusions}

The modified hemicelluloses with DS values of $0.15-0.65$ were prepared by the etherification of wheat straw hemicelluloses with acrylamide in alkaline aqueous solution. It was found that sodium hydroxide could improve the speed of this reaction remarkably and more sodium hydroxide made acylamino groups to be saponified to carboxyl groups. A DS of 0.65 was obtained with hemicelluloses in alkaline medium with 8.0 molar ratio of acrylamide to xylose unit in hemicelluloses in the presence of 1.0 molar ratio of sodium hydroxde to xylose unit in hemicelluloses at $25^{\circ} \mathrm{C}$ for $12 \mathrm{~h}$. The DS of hemicelluloses ethers significantly increased with an increase in the dosage of acrylamide applied. Because of the presence of carbamoylethyl groups and carboxyl groups, these modified hemicelluosic polymers may be used as wet end additives in papermaking, flocculation, detergents, food etc. Certainly, much more work is needed on the substituent distribution on site of $C$ atoms of the xylose unit in hemicelluloses and the carbamoylethyl groups to carboxyethyl groups ratio as well as molecular weight distribution.

\section{Experimental}

\section{Materials}

Wheat straw was obtained from the experimental farm of the North-Western University of Agricultural and Forest Sciences and Technology (Yangling, China). It was first dried in sunlight and then ground to pass a $1.2 \mathrm{~mm}$ size screen. The ground straw was further dried again in a cabinet oven with air circulation for $16 \mathrm{~h}$ at $50{ }^{\circ} \mathrm{C}$. Acrylamide (AM) and other chemicals were analytical grade and were purchased from Guangzhou Chemical Reagent Factory, China.

\section{Preparation of carbamoylethyl hemicelluloses}

For a typical synthesis, $0.66 \mathrm{~g}$ of wheat straw hemicelluloses powder isolated according to the method in a previous paper [24] $(0.01 \mathrm{~mol}$ of hydroxyl functionality in hemicelluloses) in $4 \mathrm{~mL}$ water was heated to $80^{\circ} \mathrm{C}$ under stirring until completely dissolved (approximately $10 \mathrm{~min}$ ), and then the mixture was cooled down at room temperature. A solution of $0.2 \mathrm{~g} \mathrm{NaOH}$ in $4 \mathrm{~mL}$ water was added. After 20 min at room temperature, and then acrylamide in $4 \mathrm{~mL}$ water were added to the dissolved hemicelluloses, and the mixture was allowed to react for $12 \mathrm{~h}$ under stirring at room temperature. When the reaction finished, acetic acid solution was added into the mixture solution to neutralize, and then washed with $95 \%$ ethanol. The product was 
dialyzed (cut off 3500) with distilled water, and then freeze-dried. All the etherification experiments were performed in duplicate. The standard errors or derivations were observed to be lower than $\pm 5 \%$.

\section{Characterization of hemicelluloses and their derivatives}

The neutral sugar composition of the isolated hemicelluloses was determined by gas chromatography (GC) analysis of their alditol acetates [25]. The content of uronic acids in native hemicelluloses was estimated colourometrically by the method of Blumenkrantz and Asboe-Hanson [26]. The solubility of the resulted products in distilled water was measured at $25^{\circ} \mathrm{C}$, and the concentration was about $1 \%(\mathrm{w} / \mathrm{v})$.

FT-IR spectra were obtained on an FT-IR spectrophotometer (Nicolet 510) using a $\mathrm{KBr}$ disc containing $1 \%$ finely ground samples. Thirty-two scans were taken for each sample recorded from 4000 to $400 \mathrm{~cm}^{-1}$ at a resolution of $2 \mathrm{~cm}^{-1}$ in the transmission mode. The solution-state ${ }^{13} \mathrm{C}$ NMR spectra were recorded on a Bruker DRX-400 spectrometer at $25{ }^{\circ} \mathrm{C}$ from $80 \mathrm{mg}$ of sample dissolved in $1.0 \mathrm{~mL} \mathrm{D}_{2} \mathrm{O}$ for native hemicelluloses and their derivates after 15,000 scans. A $60^{\circ}$ pulse flipping angle, a $3.9 \mu \mathrm{s}$ pulse width and a $0.85 \mathrm{~s}$ delay time between scans were used. The solutionstate ${ }^{1} \mathrm{H}$ NMR spectra were recorded on a Bruker DRX-400 spectrometer at $25{ }^{\circ} \mathrm{C}$ using $15 \mathrm{mg}$ of sample dissolved in $1.0 \mathrm{~mL} \mathrm{D}_{2} \mathrm{O}$ for native hemicelluloses and their derivates.

Thermal stability of the native and the modified hemicellulosic samples was performed using a simultaneous thermal analyzer (Pyris Diamond TG/DTA, PE Instrument). The sample weighed between 8 and $12 \mathrm{mg}$. The scans were run from room temperature to $600{ }^{\circ} \mathrm{C}$ at a rate of $10{ }^{\circ} \mathrm{C}$ per minute under nitrogen flow. Prior to thermal analysis, the samples were dried in vacuum at $80^{\circ} \mathrm{C}$ for $24 \mathrm{~h}$.

\section{Acknowledgements}

This work was supported by the grants from the Natural Science Foundation of China (No. 30430550, 30710103906), China Ministries of Education (No. 111) and Science and Technology ( 2006CD708406 ), and the Doctorate Foundation of South China University of Technology.

\section{References}

[1] Ragauska, A. J.; Williams, C. K.; Davison, B. H.; Britovsek, G.; Cairney, J.; Eckert, C. A.; Frederick, W. J.; Hallett, J. P.; Leak, D. J.; Liotta, C. L.; Mielenz, J. R.; Murphy, R.; Templer, R.; Tschaplinski, T. Science 2006, 311, 484.

[2] Heinze, T.; Koschella, A.; Ebringerova, A. ACS Symposiun Series 2004, 864, 312. [3] Barthel, S.; Heinze, T. Green Chem. 2006, 8, 301.

[4] Ebringerova, A.; Hormadkova, Z.; Kauracova, M.; Antal, M. Carbohyd. Polym. 1994, 24, 301.

[5] Schwikal, K.; Heinze, T.; Ebringerova, A.; Petzold, K. Macromol. Symp. 2006, 232, 49.

[6] Ren, J. L.; Sun, R. C.; Liu, C. F.; Chao, Z. Y.; Luo, W. Polym. Degrad. Stabil. 2006, 91, 2579.

[7] Ren, J. L.; Xu, F.; Sun, R. C.; Peng, B.; Sun, J. X. J. Agr. Food Chem. 2008, 56, 1251.

[8] Sun, X. F.; Sun, R. C.; Sun, J. X. J. Sci. Food Agr. 2004, 84, 800.

[9] Sun, R. C.; Fang, J. M.; Tomkinson, J. S. Polym. Degrad. Stabil. 2000, 67, 345. 
[10] Capek, P.; Alföldi, J.; Lišková, D. Carbohyd. Res. 2002, 337, 1033.

[11] Sun, R. C.; Lawther, J. M.; Banks, W. B. Carbohyd. Polym. 1996, 29, 325.

[12] Wilkie, K. C. B. Adv. Carbohyd. Chem. Biochem. 1979, 36, 215.

[13] Wurzburg, O. B. Application and Property of Modified Starch 1989, 238.

[14] Chen, P. H.; Zhu, Z. F. Shanghai Text. Sci. Tech. 2007, 35, 10.

[15] Song, Y. B.; Zhou, J. P.; Zhang, L.; Wu, X. Carbohyd. Polym. 2008, 73, 18.

[16] Klemm, D.; Philipp, B.; Heinze, T.; Heinze, U.; Wagenknecht, W. Comprehensive Cellulose Chemi. 1998, 2, 31.

[17] Kacurakova, M.; Belton, P. S.; Wilson, R. H.; Hirsch, J.; Ebringerova, A. J. Sci. Food Agr. 1998, 77, 38.

[18] Geng, Z. C.; Sun, R. C.; Sun, X. F.; Lu, Q. Polym. Degrad. Stabil. 2003, 80, 315. [19] Teleman, A.; Lundqvist, J.; Tjerneld, F.; Stalbrand, H.; Dahlman, O. Carbohyd. Res. 2000, 329, 807.

[20] Gabrielli, I.; Gatenholm, P.; Glasser, W. G.; Jain, R. K; Kenne, L. Carbohyd. Polym. 2000, 43, 367.

[21] Imamura, T.; Watanabe, T.; Kuwahara, M.; Koshijima, T. Phytochemistry 1994, $37,1165$.

[22] Halverson, F.; Lancaster, J. E.; O'Conner, M. N. Macromolecules 1985, 18, 1139 .

[23] Zurimendi, J. A.; Guerrero, S. J.; Leon, V. Polymer 1984, 25, 1314.

[24] Ren, J. L.; Sun, R. C.; Liu, C. F.; Cao, Z. N.; Luo, W. Carbohydr. Polym. 2007, $70,406$.

[25] Blakeney, A. B.; Harris, P. J.; Henry, R. J.; Stone, B. A. Carbohyd. Res. 1983, 113, 291.

[26] Blumenkrantz, N.; Asboe-Hanson, G. Anal. Biochem. 1973, 54, 484. 